Ciência Florestal, Santa Maria, v. 27, n. 4, p. 1339-1347, out.-dez., 2017

ISSN 1980-5098

\title{
ANÁLISE ECONÔMICA DA ROTAÇ̃̃O FLORESTAL DE POVOAMENTOS DE EUCALIPTO UTILIZANDO A SIMULAÇÃO DE MONTE CARLO
}

\author{
ECONOMIC ANALYSIS OF ROTATION FOREST OF EUCALYPTUS STANDS USING MONTE \\ CARLO SIMULATION
}

\author{
Juliana Carneiro Gonçalves ${ }^{1}$ Antônio Donizette de Oliveira ${ }^{2}$ Samuel de Pádua Chaves e Carvalho ${ }^{3}$ \\ Lucas Rezende Gomide ${ }^{2}$
}

\begin{abstract}
RESUMO
O objetivo deste trabalho foi determinar a rotação econômica de plantações de eucalipto em três diferentes sítios produtivos sob condições de risco. Os dados utilizados na realização do estudo são provenientes de plantios equiâneos e monoclonais do híbrido Eucalyptus urograndis, e cedidos pela empresa Fibria Celulose. Além dos dados de produção das áreas foram também cedidas as informações de fluxo de caixa. Para a análise de risco utilizou-se o método de Monte Carlo, tendo como resultado da simulação o Valor Presente Líquido para séries Infinitas e, como variáveis de entrada e/ou fontes de incertezas, as distribuições de probabilidade referentes ao preço da terra e da madeira, o custo de colheita e transporte, a taxa de juros e além da produção de madeira. A simulação constituiu na realização 10.000 de iterações, em que foram obtidas as informações necessárias ao prosseguimento das análises. Concluiu-se que a rotação ótima para corte foi de 6, 8 e 11 anos nos sítios bom (I), médio (II) e ruim (III), respectivamente, sendo, portanto, condizente com as leis biológicas de crescimento. Conclui-se ainda que sob as mesmas condições de manejo, sítios mais produtivos geram maior retorno econômico e por consequência menor risco em cenários econômicos desfavoráveis.
\end{abstract}

Palavras-chave: economia florestal; simulação de Monte Carlo; eucalipto.

\begin{abstract}
The objective of this study was to determine the economic cycle rotation of eucalyptus plantations in three productivity sites over hazardous conditions. The data used on this study, released by the company Fibria Celulose. Besides the timber production data, it was also given the company cash flow. It was from an evenaged stand and a monoclonal stand both of Eucalyptus urograndis. For the risk analysis, the study used Monte Carlo method, having as a result of the simulation the Net Present Value for Infinite Series, using as an input variable and/or source of uncertainty, the probability distributions of the land and wood prices, the costs of harvesting and transportation activities, the interest rate and also the timber production. The simulation consisted in carrying out 10,000 interactions, where it was obtained the necessary information for the development of the next analysis. As a conclusion the optimal rotation for cutting was 6,8 and 11 years on the good sites (I), regular sites(II) and poor sites(III) respectively, being consistent with the biological laws of growth. Another conclusion is that even under the same management conditions, more productive sites generate greater economic returns and therefore lower risk in adverse economic scenarios. Keywords: forest economics; Monte Carlo simulation; Eucalyptus.

1 Engenheira Florestal, MSc., Av. Oito de Dezembro, 625, CM102, CEP 36307-250, São João del-Rei (MG), Brasil. jujacarneiro@gmail.com

2 Engenheiro Florestal, Dr., Professor do Departamento de Ciências Florestais, Universidade Federal de Lavras, Campus universitário, Caixa Postal 3037, CEP 37200-000, Lavras (MG), Brasil. donizete@dcf.ufla.br / lucasgomide@dcf.ufla.br

3 Engenheiro Florestal, Dr., Professor da Faculdade de Engenharia Florestal, Universidade Federal do Mato Grosso, Av. Fernando Corrêa da Costa, 2367, CEP 78060-900, Cuiabá (MT), Brasil. sam.padua@gmail.com
\end{abstract}




\section{INTRODUÇÃO}

O setor florestal brasileiro tem tido grande destaque na economia nacional, atraindo muitos investimentos em pesquisa e produção (REZENDE; OLIVEIRA; RODRIGUES, 2005). Entre as espécies florestais utilizadas nesse setor destaca-se o eucalipto, que apresenta rápido crescimento, potencial para grande número de sortimentos e adaptabilidade a várias regiões do país. Sendo o gênero florestal com maior área plantada no Brasil e devido a seu rápido crescimento, a definição da rotação florestal torna-se de suma importância. Segundo Resende et al. (2004), a rotação florestal nada mais é do que a idade de corte de um povoamento e consiste em uma das fases mais importantes do planejamento e manejo florestal no qual o principal objetivo do empreendimento é a maximização do lucro.

Rezende, Oliveira e Coelho Junior (2005) citam que os tipos de rotações mais conhecidas são a ecológica ou física, a silvicultural, a técnica, a de máxima produtividade volumétrica e a econômica. Esses autores afirmam que a definição da idade ótima é afetada diretamente por fatores técnicos como espaçamento, sítio, produtos desejados, bem como por fatores econômicos como o preço da madeira, custos de produção, taxa de desconto, valor da terra, e horizonte de planejamento. Complementar a estas informações podem ser citados outros fatores como incêndios, presença de pragas e doenças, e eventos naturais.

A rotação econômica de um povoamento florestal é determinada pela estimativa do seu fluxo de caixa, ou seja, informações de custos e receitas compiladas em um período de tempo determinado. Contudo, esse fluxo de caixa possui as mais diversas incertezas, como, por exemplo, àquelas relacionadas às informações de custo ou receita. Como abordado por Lapponi (2007), a incerteza está relacionada com o desvio do fluxo de caixa esperado de um projeto.

Uma maneira de minimizar os efeitos das incertezas em ferramentas de tomadas de decisão é por meio de métodos de análise que permitem mensurá-las fornecendo não apenas um resultado, mas um conjunto de possíveis cenários, cada um associado a uma probabilidade de ocorrência. Segundo Mendes e Souza (2007), o grau de incerteza a respeito de um evento pode ser chamado de risco, e a análise quantitativa do risco, usando a simulação de Monte Carlo, oferece ao usuário um método consistente e preciso para abordar as mais diversas incertezas associadas às atividades de um empreendimento, como os florestais por exemplo.

No cenário econômico mundial atual, em que a incerteza faz-se presente em todos os setores da economia, a possibilidade de avaliação de como o comportamento das variáveis pode afetar o resultado de projetos, torna-se determinante para o sucesso futuro da organização (CARDOSO; AMARAL, 2000). Sendo assim, a abordagem central deste estudo foi a determinação da rotação econômica de plantações clonais de eucalipto em condições de risco em três distintos sítios produtivos.

\section{MATERIAIS E MÉTODOS}

\section{Caracterização e análise dos dados}

Aárea de estudo é constituída de plantios monoclonais de eucalipto situados em três sítios diferentes, que pertencem à empresa Fibria Celulose S.A., cuja sede é no município de Aracruz, Espírito Santo, Brasil. A temperatura média da região é de $28^{\circ} \mathrm{C}$ e a precipitação anual média é de $1.200 \mathrm{~mm}$. Os tipos de solo predominantes na região são o Latossolo Vermelho Amarelo Distrófico e Podzólico Vermelho Amarelo (PREFEITURA MUNICIPAL DE ARACRUZ, 2013). Foram utilizados dados de plantios de híbridos de Eucalyptus grandis x Eucalyptus urophylla históricos e ativos que se encontravam a partir do segundo ciclo, com referência em dezembro de 2012.

Para determinar a rotação econômica dos plantios de eucalipto foram utilizadas informações sobre o crescimento e a produção volumétrica de madeira dos plantios monoclonais do trabalho de Gonçalves (2014) apresentados na Tabela 1, assim, como informações sobre as receitas e os custos de produção de madeira desses plantios, informações estas que foram cedidas pela empresa Fibria. 
TABELA 1: Volume de madeira $\left(\mathrm{m}^{3}\right)$ para os diferentes sítios e idades.

TABLE 1: Wood volume $\left(\mathrm{m}^{3}\right)$ for different sites and ages.

\begin{tabular}{cccc}
\hline \multirow{2}{*}{ Idade } & \multicolumn{3}{c}{ Produtividade $\left(\mathrm{m}^{3} \mathrm{ha}^{-1}\right)$} \\
\cline { 2 - 4 } & Sítio I & Sítio II & Sítio III \\
\hline 1 & 21,17 & 19,10 & 15,31 \\
2 & 56,36 & 43,43 & 30,82 \\
3 & 112,42 & 82,11 & 49,49 \\
4 & 166,30 & 125,16 & 72,51 \\
5 & 207,58 & 164,15 & 97,98 \\
6 & 236,69 & 195,42 & 123,68 \\
7 & 257,08 & 219,10 & 147,76 \\
8 & 271,77 & 236,74 & 169,13 \\
9 & 282,75 & 249,97 & 187,39 \\
10 & 291,28 & 260,05 & 202,62 \\
11 & 298,09 & 267,88 & 215,16 \\
12 & 303,68 & 274,08 & 225,41 \\
13 & 308,34 & 279,07 & 233,78 \\
14 & 312,27 & 283,16 & 240,63 \\
15 & 315,61 & 286,54 & 246,24 \\
\hline
\end{tabular}

\section{Custos e receitas inerentes aos plantios avaliados}

Para determinar a rotação econômica dos plantios florestais foram disponibilizados pela empresa, os custos médios relacionados às atividades de implantação e manutenção das áreas conforme descritos na Tabela 2.

Como custo anual da terra foram considerados os juros sobre o seu valor, sendo que o valor adotado foi de $\mathrm{R} \$ 8.000,00$ por hectare. A taxa real de juros utilizada na análise econômica foi de $3 \%$ ao ano.

A receita foi obtida multiplicando-se o volume de madeira colhido pelo preço de venda. Como a empresa não vende esse produto, mas o utiliza como matéria-prima em seu processo industrial, foi considerado o preço de $\mathrm{R} \$ 80,00$ por metro cúbico colocado no pátio de estocagem, que é o valor vigente na região.

\section{Determinação da rotação econômica em condições de risco}

Para determinar a rotação econômica em condições de risco foi utilizada a simulação de Monte Carlo, que se inicia pela construção do modelo-base necessário para determinar o indicador a ser utilizado na análise econômica. Esse modelo-base foi o fluxo de caixa construído usando as estimativas dos custos e receitas que ocorreram durante o horizonte de planejamento estabelecido para a análise. Para isso fez-se necessário determinar as variáveis de entrada e saída do modelo apresentadas a seguir.

\section{Variáveis de saída (output)}

A variável de saída do modelo (output) é o resultado da simulação a partir da qual é feita a tomada de decisões em relação à viabilidade econômica do plantio de eucalipto nos três diferentes sítios e à determinação de sua rotação econômica, em condições de risco. Assim, o Valor Presente Líquido para o horizonte de planejamento infinito ( $\mathrm{VPL}_{\infty}$ ) foi o indicador econômico utilizado. Segundo Rezende e Oliveira (2008), ele é calculado pela seguinte fórmula, 


$$
V P L_{\infty}=\frac{V P L(1+i)^{n}}{(1+i)^{n}-1}
$$

Em que:

$$
V P L=\sum_{j=0}^{n} R_{j}(1+i)^{-j}-C_{j}(1+i)^{-j}
$$

Em que: $\mathrm{Cj}=$ custo no final do ano $\mathrm{j} ; \mathrm{Cj}=$ receita no final do ano $\mathrm{j} ; \mathrm{n}=$ idade de corte do povoamento florestal; $i=$ taxa real de juros.

\section{Variáveis de entrada (input)}

O passo seguinte foi a definição das variáveis de entrada, ou variáveis de risco, ou variáveis incertas do modelo-base. Nesse caso, a suposição é de que o fluxo de caixa é incerto, ou seja, as estimativas dos custos e receitas relacionados ao plantio de eucalipto são consideradas uma fonte de riscos, pois existem incertezas em relação a esses valores. Assim, sendo o $\mathrm{VPL}_{\infty}$ uma estimativa que depende desses valores, quanto maior for a incerteza dos mesmos, tanto maior será a incerteza do VPL . $_{\infty}$

As variáveis de risco consideradas no estudo foram a taxa de juros, o custo da terra, o custo de colheita e transporte (custo caixa da madeira), o preço de venda da madeira e a produção volumétrica de madeira. Para representar o comportamento incerto dessas variáveis foi utilizada a distribuição de probabilidade triangular que consiste em definir valores mínimos, mais prováveis e máximos para cada uma delas (Tabela 3). Segundo Cardoso e Amaral (2000), uma das desvantagens da aplicação da simulação de Monte Carlo é a dificuldade em se definir os formatos das distribuições de probabilidades das variáveis que melhor representem a realidade. Souza (2001) considera que as distribuições triangular e uniforme, geralmente, são muito utilizadas nas ciências agrárias e na economia, por apresentarem simplicidade e a vantagem de não necessitarem de muitos dados de um determinado evento.

Assim como as informações médias de produção (Tabela 2), as informações inerentes aos custos praticados em cada atividade da empresa foram também cedidas pela Fibria e, deste modo, praticadas no momento de elaboração do estudo.

TABELA 3: Valores mais prováveis, mínimos e máximos das variáveis de risco.

TABLE 3: Most likely values, minimum and maximum of the risk variables.

\begin{tabular}{lccc}
\hline \multicolumn{1}{c}{ Variável } & Mínimo & Mais provável & Máximo \\
\hline Custo Colheita & 17,10 & 19,00 & 20,90 \\
Custo Tr. Madeira & 27,90 & 31,00 & 34,10 \\
Valor terra & $6.400,00$ & $8.000,00$ & $9.600,00$ \\
Preço mad. & 72,00 & 80,00 & 88,00 \\
Produtividade & $\mathrm{x}-10 \%$ & $\mathrm{x}$ & $\mathrm{x}+10 \%$ \\
Taxa de Juros & $2 \%$ & $3 \%$ & $4 \%$ \\
\hline
\end{tabular}

\section{Simulação e análise de dados}

A simulação foi realizada com o auxílio do software@RISK (PALISADE CORPORATION, 2009). Primeiramente, foi realizada uma simulação automática pelo software, visando monitorar a convergência. Em seguida, realizou-se uma segunda simulação com 10.000 iterações, número este acima daquele em que 
houve convergência. A partir das iterações realizadas, foram gerados os resultados relacionados ao $\mathrm{VPL}_{\infty}$, como distribuições de probabilidade, frequências acumulada e relativa, estatísticas descritivas.

\section{RESULTADOS E DISCUSSÕES}

\section{Análise de risco}

De acordo com os resultados da Tabela 4 foi possível notar que o maior valor de VPL $\infty$ está associado ao sítio I e ocorre aos 6 anos ( $\mathrm{R} \$ 11.253,62 / \mathrm{ha}$ ), indicando essa idade como sendo a ótima de corte ou rotação econômica. Quando analisados os percentis observa-se que $5 \%$ dos valores de VPL $\infty$ são superiores a $\mathrm{R} \$ 23.218,00 /$ ha e $5 \%$ são inferiores a $\mathrm{R} \$ 1.374,54 /$ ha, havendo assim, apenas $2,6 \%$ de probabilidade de ocorrência de valores negativos para esse indicador econômico.

No sítio II, o maior valor de VPL $\infty$ ocorreu aos 8 anos (R $\$ 3.999,87 / \mathrm{ha})$, sendo essa a idade ótima de corte para este sítio. Quando analisados os percentis observa-se que 5\% dos valores de VPL $\infty$ são superiores a $\mathrm{R} \$ 12.638,59 /$ ha e 5\% são inferiores a R $\$-3.168,96$ /ha, havendo assim, 3,4\% de probabilidade de ocorrência de valores negativos para o VPL $\infty$.

O sítio III apresentou VPL $\infty$ negativo em todas as idades, portanto, a rotação econômica é a idade de menor VPL $\infty$ negativo, ou seja, 11 anos (R \$-2.160,28/ha). A princípio, esse resultado indica que plantar eucalipto em sítios com essa qualidade é inviável economicamente. Porém, a avaliação sob condições de risco é relativa e em determinadas condições pode-se obter viabilidade econômica para esse sítio. Por exemplo, na idade de 11 anos, há $72,7 \%$ de probabilidade de que os valores de VPL $\infty$ sejam negativos. Assim, dependendo da combinação das variáveis de entrada, existem $27,3 \%$ de chance de que os VPL $\infty$ restantes sejam positivos, ou seja, há chances de se obter lucro plantando eucalipto nesse sítio.

Assim, como já era esperada, a rotação econômica tende a ser inversamente proporcional à produtividade dos sítios, ou seja, quanto maior a produtividade do sítio, menor a rotação econômica. Segundo Gomes et al. (2002), isso ocorre porque, nos melhores sítios, a idade de máximo IMA (Incremento Médio Anual) ocorre mais cedo do que nos sítios menos produtivos, o que faz com que a idade ótima de corte dos sítios mais produtivos seja inferior quando comparada à idade ótima dos sítios menos produtivos, ao passo que nestes sítios, a floresta demanda um período de tempo superior para alcançar o máximo IMA.

Observa-se, que nem sempre a idade ótima de corte é a idade de menor risco. A menor probabilidade de apresentar valores negativos foi apresentada pelo sítio I aos 7 anos de idade $(2,4 \%)$. Já no sítio II isso ocorreu aos 7 anos (2,4\%) e no sítio III aos 10 e 11 anos (72,7\%). Segundo Hacura, Jamadus-Hacura e Kotot (2001), o projeto é considerado de baixo risco quando a probabilidade de ocorrência de VPLs negativos é inferior a 20\%. Nessas condições, o plantio de eucalipto só pode ser considerado como sendo de baixo risco nos sítios I e II. Entretanto, segundo Silva (2011), o risco é uma variável subjetiva, na qual cada gestor tem uma medida de aversão sobre este.

Assim, a análise de risco fornece um número maior de informações ao tomador de decisão, possibilitando ao mesmo vislumbrar o impacto no VPL ao se tomar uma decisão mais ou menos conservadora. Por exemplo, no sítio II, a diferença de risco entre a idade de maior VPL (8 anos) e a de menor risco (7 anos) é de $1 \%$ e a redução do VPL é de apenas $\mathrm{R} \$ 20,96$ ha-1 cabendo ao tomador de decisão escolher o que é mais interessante. Já no sítio III, observa-se que o risco é o mesmo quando o corte da floresta é realizado aos 10 ou 11 anos, optando-se então pelo corte na idade de menor prejuízo. Segundo Silva et al. (2011), a análise de risco propicia a obtenção de informações adicionais às obtidas pela análise determinística, facilitando a compreensão e aumentando a segurança para a tomada de decisão em relação à viabilidade econômica de investimentos.

Na idade em que ocorre a rotação econômica do sítio II (8 anos), o VPL $\infty$ esperado é de 3.999,87e o desvio padrão é de 4.860,37. Assim, uma diminuição equivalente a menos de um desvio padrão no VPL $\infty$ é suficiente para que nesse sítio os plantios de eucalipto se inviabilizem economicamente, já que nessas condições esse indicador econômico seria negativo. Por outro lado, no sítio I é preciso haver variação de mais 1,5 desvios padrão no VPL $\infty$ para que o mesmo seja negativo, mostrando que nesse sítio os plantios estão sujeitos a menos risco que no sítio II.

Silva (2011) analisou a viabilidade econômica e a rotação econômica de plantios de candeia 
TABELA 4: Estatísticas descritivas do $\mathrm{VPL}_{\infty}$ para as diferentes idades de corte nos sítios I, II e III. TABLE 4: Descriptive statistics of NPV $\infty$ for different cutting ages at sites I, II and III.

\begin{tabular}{|c|c|c|c|c|c|c|c|c|}
\hline \multicolumn{9}{|c|}{ Sítio I } \\
\hline Idade & $\begin{array}{c}\text { VPL } \infty \text { esperado } \\
\left(\mathrm{R} \$ \cdot \mathrm{ha}^{-1}\right)\end{array}$ & $\begin{array}{l}\text { Mínimo } \\
\left(\mathrm{R} \$ . h^{-1}\right)\end{array}$ & $\begin{array}{l}\text { Máximo } \\
\left(\mathrm{R} \$ . \mathrm{ha}^{-1}\right)\end{array}$ & $\begin{array}{l}\text { Desvio padrão } \\
\quad\left(\mathrm{R} \$ \cdot \mathrm{ha}^{-1}\right)\end{array}$ & $\mathrm{VPL} \infty<0$ & $\begin{array}{c}5 \% \\
\left(\mathrm{R} \$ . \mathrm{ha}^{-1}\right)\end{array}$ & $\begin{array}{c}50 \% \\
\left(\mathrm{R} \$ . \mathrm{ha}^{-1}\right)\end{array}$ & $\begin{array}{c}95 \% \\
\left(\mathrm{R} \$ . \mathrm{ha}^{-1}\right)\end{array}$ \\
\hline 3 & $-13.660,80$ & $-32.731,11$ & $9.090,59$ & $5.445,28$ & $99,3 \%$ & $-22.466,63$ & $-13.736,06$ & $-4.513,78$ \\
\hline 4 & $2.925,92$ & $-14.352,33$ & $35.141,20$ & $6.482,39$ & $34,2 \%$ & $-6.963,06$ & $2.478,26$ & $14.348,92$ \\
\hline 5 & $9.595,85$ & $-8.148,20$ & $40.270,43$ & $6.835,42$ & $6,4 \%$ & $-577,06$ & $8.984,60$ & $21.763,19$ \\
\hline 6 & $11.253,62$ & $-6.733,70$ & $47.338,54$ & $6.708,61$ & $2,6 \%$ & $1.374,54$ & $10.660,90$ & $23.218,00$ \\
\hline 7 & $10.729,45$ & $-5.252,60$ & $43.469,65$ & $6.308,16$ & $2,4 \%$ & $1.470,28$ & $10.073,81$ & $22.083,88$ \\
\hline 8 & $9.383,65$ & $-6.816,73$ & $38.176,65$ & $5.903,65$ & $3,4 \%$ & 672,55 & $8.829,72$ & $20.086,31$ \\
\hline 9 & $7.770,97$ & $-6.104,49$ & $32.059,86$ & $5.433,63$ & $5,4 \%$ & $-152,43$ & $7.219,52$ & $17.513,65$ \\
\hline 10 & $6.159,51$ & $-6.502,85$ & $35.368,09$ & $5.043,55$ & $9,5 \%$ & $-1.143,78$ & $5.641,77$ & $15.320,89$ \\
\hline 11 & $4.630,04$ & $-8.042,36$ & $27.127,56$ & $4.665,34$ & $15,5 \%$ & $-2.201,50$ & $4.153,72$ & $13.149,34$ \\
\hline 12 & $3.222,89$ & $-7.457,28$ & $27.347,79$ & $4.350,65$ & $24,3 \%$ & $-3.145,55$ & $2.777,70$ & $11.115,57$ \\
\hline 13 & $1.936,93$ & $-8.487,50$ & $22.596,37$ & $4.045,33$ & $34,7 \%$ & $-3.935,58$ & $1.490,75$ & $9.270,98$ \\
\hline 14 & 771,86 & $-8.198,02$ & $19.707,88$ & $3.805,49$ & $46,2 \%$ & $-4.675,80$ & 371,10 & $7.719,02$ \\
\hline 15 & $-293,11$ & $-9.987,97$ & $17.803,42$ & $3.547,48$ & $57,4 \%$ & $-5.411,56$ & $-698,12$ & $6.231,06$ \\
\hline \multicolumn{9}{|c|}{ Sítio II } \\
\hline Idade & $\begin{array}{l}\text { VPL } \infty \text { esperado } \\
\left(\mathrm{R} \$ . \mathrm{ha}^{-1}\right)\end{array}$ & $\begin{array}{l}\text { Mínimo } \\
\left(\mathrm{R} \$ . \mathrm{ha}^{-1}\right)\end{array}$ & $\begin{array}{l}\text { Máximo } \\
\left(\mathrm{R} \$ \cdot \mathrm{ha}^{-1}\right)\end{array}$ & $\begin{array}{l}\text { Desvio padrão } \\
\quad\left(\mathrm{R} \$ \cdot \mathrm{ha}^{-1}\right)\end{array}$ & VPL $\infty<0$ & $\begin{array}{c}5 \% \\
\left(\mathrm{R} \$ \cdot \mathrm{ha}^{-1}\right)\end{array}$ & $\begin{array}{c}50 \% \\
\left(\mathrm{R} \$ \cdot \mathrm{ha}^{-1}\right)\end{array}$ & $\begin{array}{c}95 \% \\
\left(\mathrm{R} \$ . \mathrm{ha}^{-1}\right)\end{array}$ \\
\hline 3 & $-27.013,33$ & $-44.979,30$ & $-13.346,28$ & $4.467,51$ & $99,6 \%$ & $-34.819,12$ & $-26.771,97$ & $-20.041,37$ \\
\hline 4 & $-10.465,95$ & $-24.942,54$ & $8.204,52$ & $4.485,06$ & $35,6 \%$ & $-17.638,14$ & $-10.572,02$ & $-2.900,76$ \\
\hline 5 & $-1.549,59$ & $-15.404,64$ & $24.153,76$ & $4.951,21$ & $6,7 \%$ & $-9.130,43$ & $-1.886,35$ & $7.106,21$ \\
\hline 6 & $2.559,41$ & $-11.982,14$ & $28.437,83$ & $5.174,73$ & $2,9 \%$ & $-5.207,53$ & $2.151,81$ & $11.702,26$ \\
\hline 7 & $3.978,92$ & $-9.506,48$ & $27.556,52$ & $5.122,96$ & $2,4 \%$ & $-3.636,09$ & $3.542,32$ & $13.101,86$ \\
\hline 8 & $3.999,88$ & $-8.813,56$ & $27.125,58$ & $4.860,37$ & $3,4 \%$ & $-3.168,96$ & $3.582,91$ & $12.638,59$ \\
\hline 9 & $3.367,46$ & $-8.005,55$ & $23.813,98$ & $4.621,01$ & $5,6 \%$ & $-3.433,66$ & $2.956,29$ & $11.733,64$ \\
\hline 10 & $2.444,29$ & $-9.126,33$ & $21.884,15$ & $4.380,88$ & $9,7 \%$ & $-3.916,21$ & $1.975,30$ & $10.481,00$ \\
\hline 11 & $1.409,34$ & $-9.099,00$ & $21.372,91$ & $4.072,09$ & $15,7 \%$ & $-4.593,40$ & $1.031,49$ & $8.701,75$ \\
\hline 12 & 373,66 & $-9.243,64$ & $20.208,72$ & $3.824,57$ & $23,7 \%$ & $-5.200,36$ & 9,39 & $7.288,68$ \\
\hline 13 & $-626,36$ & $-10.133,86$ & $18.367,76$ & $3.568,27$ & $34,7 \%$ & $-5.845,07$ & $-1.001,45$ & $5.882,83$ \\
\hline 14 & $-1.561,37$ & $-10.700,52$ & $14.739,67$ & $3.365,61$ & $45,9 \%$ & $-6.456,96$ & $-1.937,21$ & $4.483,70$ \\
\hline 15 & $-2.431,07$ & $-10.705,21$ & $11.363,51$ & $3.174,18$ & $57,3 \%$ & $-7.082,86$ & $-2.759,32$ & $3.384,69$ \\
\hline \multicolumn{9}{|c|}{ Sítio III } \\
\hline Idade & $\begin{array}{l}\text { VPL } \infty \text { esperado } \\
\left(\mathrm{R} \$ \mathrm{ha}^{-1}\right)\end{array}$ & $\begin{array}{l}\text { Mínimo } \\
\left(\mathrm{R} \$ \cdot \mathrm{ha}^{-1}\right)\end{array}$ & $\begin{array}{l}\text { Máximo } \\
\left(\mathrm{R} \$ \cdot \mathrm{ha}^{-1}\right)\end{array}$ & $\begin{array}{l}\text { Desvio padrão } \\
\quad\left(\mathrm{R} \$ \cdot \mathrm{ha}^{-1}\right)\end{array}$ & VPL $\infty<0$ & $\begin{array}{c}5 \% \\
\left(\mathrm{R} \$ \cdot \mathrm{ha}^{-1}\right)\end{array}$ & $\begin{array}{c}50 \% \\
\left(\mathrm{R} \$ . \mathrm{ha}^{-1}\right)\end{array}$ & $\begin{array}{c}95 \% \\
\left(\mathrm{R} \$ . \mathrm{ha}^{-1}\right)\end{array}$ \\
\hline 3 & $-39.397,86$ & $-60.534,08$ & $-25.125,43$ & $5.144,50$ & $100,0 \%$ & $-48.700,04$ & $-38.923,01$ & $-31.785,57$ \\
\hline 4 & $-25.491,12$ & $-39.650,81$ & $-12.831,42$ & $4.087,45$ & $100,0 \%$ & $-32.308,64$ & $-25.400,44$ & $-18.871,07$ \\
\hline 5 & $-16.292,36$ & $-30.471,99$ & $-2.250,38$ & $3.926,61$ & $100,0 \%$ & $-22.426,77$ & $-16.553,16$ & $-9.578,16$ \\
\hline 6 & $-10.256,37$ & $-22.366,50$ & $5.692,47$ & $4.051,99$ & $98,9 \%$ & $-16.212,01$ & $-10.626,08$ & $-3.145,19$ \\
\hline 7 & $-6.405,88$ & $-17.372,63$ & $10.771,16$ & $4.168,31$ & $92,4 \%$ & $-12.595,00$ & $-6.858,31$ & $1.004,61$ \\
\hline 8 & $-4.077,61$ & $-14.816,29$ & $17.025,73$ & $4.267,32$ & $82,6 \%$ & $-10.297,91$ & $-4.529,06$ & $3.596,02$ \\
\hline 9 & $-2.812,16$ & $-13.046,39$ & $14.965,01$ & $4.229,96$ & $75,9 \%$ & $-8.886,83$ & $-3.316,47$ & $4.912,54$ \\
\hline 10 & $-2.251,63$ & $-12.650,18$ & $15.307,02$ & $4.158,67$ & $72,7 \%$ & $-8.221,85$ & $-2.704,70$ & $5.382,62$ \\
\hline 11 & $-2.160,28$ & $-11.985,19$ & $14.063,40$ & $3.990,11$ & $72,7 \%$ & $-7.918,30$ & $-2.615,57$ & $5.056,13$ \\
\hline 12 & $-2.353,78$ & $-11.612,28$ & $15.149,68$ & $3.831,39$ & $74,5 \%$ & $-7.873,05$ & $-2.801,55$ & $4.705,13$ \\
\hline 13 & $-2.720,54$ & $-11.670,52$ & $13.272,32$ & $3.668,26$ & $78,2 \%$ & $-7.977,72$ & $-3.180,60$ & $3.997,75$ \\
\hline 14 & $-3.187,62$ & $-11.678,55$ & $11.144,13$ & $3.492,11$ & $81,9 \%$ & $-8.227,70$ & $-3.590,66$ & $3.137,90$ \\
\hline 15 & $-3.704,58$ & $-11.904,93$ & $10.829,95$ & $3.320,92$ & $86,0 \%$ & $-8.440,31$ & $-4.090,75$ & $2.304,39$ \\
\hline
\end{tabular}

Em que: VPL $\infty$ = Valor Presente Líquido para o horizonte de planejamento infinito. 
(Eremanthus erythropappus) em diversos espaçamentos, sob condições determinísticas e de risco. Para tanto, o autor utilizou o método do valor anual equivalente (VAE) tanto na análise determinística quanto na análise de risco, sendo que para esta última análise foi utilizada a simulação de Monte Carlo. $\mathrm{O}$ autor observou que o plantio de candeia em espaçamentos maiores é mais lucrativo e está sujeito a menos risco que o plantio em espaçamentos menores.

A grande vantagem do uso da simulação de Monte Carlo reside na possibilidade de vislumbrar qual será a rotação econômica frente a variações nas variáveis de risco. Ao invés de traçar a estratégia de cortar a floresta em determinada idade confiando cegamente em um valor fechado, único, o tomador de decisão passa a ter melhores subsídios para escolher a melhor estratégia de corte.

Na Figura 1, observa-se a distribuição de probabilidades, a curva de frequência acumulada (eixo da direita), a probabilidade de ocorrerem valores positivos e negativos de VPL $\infty$ nas idades correspondentes à rotação econômica de cada sítio e à posição em que se situam os valores de VPL $\infty$ médio, moda e mediana. Observa-se, que os valores de VPL $\infty$ estão distribuídos simetricamente ao redor da média, com valores médios iguais ou muito próximos à mediana, indicando que não há inclinação acentuada da distribuição dos valores para nenhum dos lados em nenhum dos sítios, características essas, semelhantes à distribuição normal.
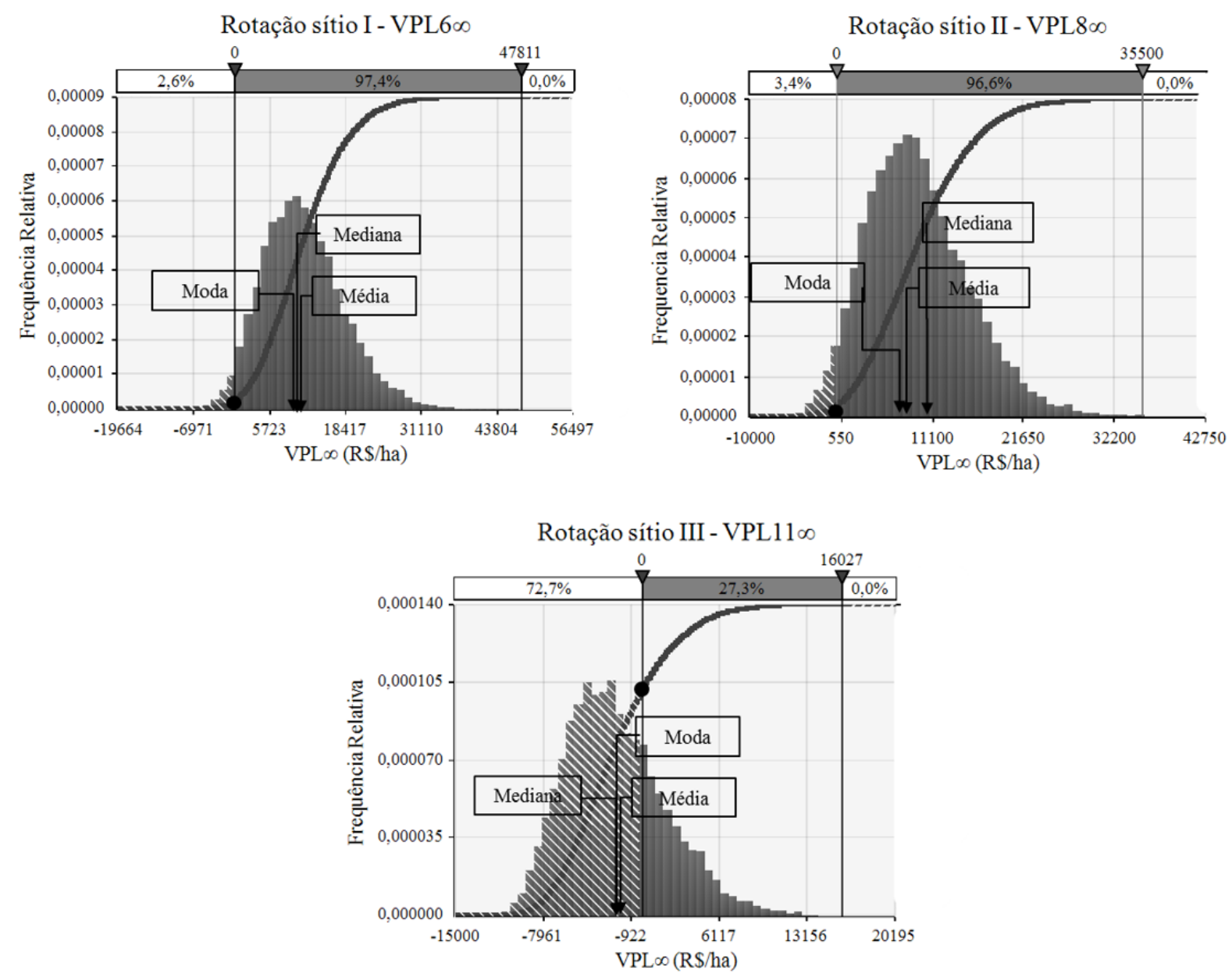

FIGURA 1: Distribuição de frequência relativa e acumulada, probabilidade de ocorrência de VPLs $_{\infty}$ positivos e negativos na rotação econômica de cada sítio, posição da média, mediana e moda dos VPLs $s_{\infty}$.

FIGURE 1: Distribution and cumulative relative frequency, probability of positive and negative NPVs $\infty$ the economic rotation of each site, position of the mean, median and mode of NPVs $\infty$. 
acentuada será a inclinação da distribuição de frequências do VPL de forma que, se o valor esperado for maior que a mediana, a distribuição terá inclinação à direita e vice-versa.

O círculo na curva de frequência acumulada em cada figura indica a interseção entre o VPL $\infty$ igual a zero e sua probabilidade de ocorrência na curva de frequência acumulada. A informação fornecida por esta interseção é a de que, no sítio I, por exemplo, na idade ótima de corte, existem 97,4\% de chances de o VPL $\infty$ ser maior que zero (frequência acumulada ao lado direito do círculo) e, consequentemente, 2,6\% de chances de o VPL $\infty$ ser menor que zero (frequência acumulada ao lado esquerdo do círculo).

Castro et al. (2007) avaliaram a viabilidade econômica da produção de carvão vegetal a partir de florestas plantadas de eucalipto em condições determinísticas e de risco utilizando o método de Monte Carlo. A análise determinística indicou o projeto como viável economicamente, apresentado VPL positivo, entretanto, a análise de risco, mostrou que o empreendimento apresentava $12 \%$ de chance de se obter valores negativos, ou seja, $12 \%$ de chance de o projeto ser inviável economicamente, proporcionando maior segurança na inferência sobre os indicadores econômicos.

Guedes et al. (2011) analisaram a viabilidade econômica da reforma e da condução da brotação de povoamentos de eucalipto, em condições determinísticas e de risco. Por meio da análise probabilística, realizada pelo método de Monte Carlo, os autores observaram que nas diversas opções de reforma e talhadia estudadas, ocorreu sempre viabilidade econômica, diminuindo as incertezas e, consequentemente, aumentando a segurança para a tomada de decisões.

Portanto, a precisão da tomada de decisão é em função do grau de domínio e conhecimento sobre as condições do mercado apresentado pelo gestor, o que torna de suma importância a disponibilidade de informações confiáveis. Se, com base no método de Monte Carlo, as decisões tomadas apresentarem probabilidade maior que $50 \%$, essas decisões já serão superiores àquelas tomadas com base nos métodos tradicionais de avaliação econômica (COELHO JUNIOR et al., 2008).

\section{CONCLUSÕES}

O método de Monte Carlo foi considerado como uma ferramenta eficiente e eficaz quando aplicado na análise de rotações de plantações florestais com eucalipto para produção de celulose sob condições de incertezas e, portanto, complementam as decisões econômicas acerca das plantações florestais.

A idade de rotação econômica foi de 6,8 e 11 anos para os sítios bom, médio e ruim respectivamente o que condiz com as leis biológicas de crescimento. Assim, sítios mais produtivos sob mesmas condições de manejo, quando comparados aos menos produtivos tendem a estabilizar o crescimento mais precocemente e por consequência devem ser cortados neste momento. Complementarmente os sítios mais produtivos são também menos susceptíveis às condições econômicas desfavoráveis quando comparado aos de média e baixa produtividade.

\section{REFERÊNCIAS}

CARDOSO, D.; AMARAL, H. F. O uso da simulação de Monte Carlo na elaboração do fluxo de caixa empresarial: uma proposta para quantificação das incertezas ambientais. In: ENCONTRO NACIONAL DE ENGENHARIA DE PRODUÇÃO, 2000. Anais... [s. 1.: s. n.], 2000.

CASTRO, R. R. et al. Rentabilidade econômica e risco na produção de carvão vegetal. Cerne, Lavras, v. 13, n. 4 , p. $353-359,2007$.

COELHO JÚNIOR, L. M. et al. Análise de investimento de um sistema agroflorestal sob situação de risco.

Cerne, Lavras, v. 14, n. 4, p. 368-378, 2008.

GUEDES, I. C. L. et al. Economic analysis of replacement regeneration and coppice regeneration in eucalyptus stands under risk conditions. Cerne, Lavras, v. 17, n. 3, p. 393-401, 2011.

GOMES, F. S. et al. Efeitos do sítio e de cenários de custos e preços na análise de regimes de manejo com e sem desbaste em pinus taeda L. Cerne, Lavras, v. 8, n. 1, p. 13-31, 2002.

GONÇALVES, J. C. Análise de risco aplicada à determinação da rotação econômica. 2014. 89 f. Dissertação (Mestrado em Engenharia Florestal) - Universidade Federal de Lavras, Lavras, 2014. 
HACURA, A.; JADAMUS-HACURA, M.; KOCOT, A. Risk analysis in investment appraisal based on the Monte Carlo simulation technique. European Physical Journal, Les Ulis, v. 20, n. 4, p. 551-553, 2001. LAPPONI, J. C. Projetos de investimentos na empresa. Rio de Janeiro: Elsevier, 2007. 332 p.

MENDES, M. H.; SOUZA, R. C. Análise quantitativa de risco: um guia para modelagem pela simulação de Monte Carlo. Rio de Janeiro: PUC-RJ, 2007. 35 p.

PALISADE CORPORATION. @Risk: risk analysis add-in for Excel. Version 5.5. New York: Palisade Corporation, 2009.

PREFEITURA MUNICIPAL DE ARACRUZ. [Website]. [2013]. Disponível em: <http://www.pma.es.gov. br/>. Acesso em: 20 fev. 2013.

RESENDE, R. R. et al. Emprego de um modelo de crescimento e produção para determinação da rotação em povoamentos de eucalipto. Revista Árvore, Viçosa, MG, v. 28, n. 2, p. 219-225, 2004.

REZENDE, J. L. P.; OLIVEIRA, A. D. Análise econômica e social de projetos florestais. Viçosa, MG: UFV, 2008. 386 p.

REZENDE, J. L. P.; OLIVEIRA, A. D.; COELHO JUNIOR, L. M. Infinite planning horizon, land opportunity cost and Faustmann methodology. Cerne, Lavras, v. 11, n. 2, p. 101-112, 2005.

REZENDE, J. L. P.; OLIVEIRA, A. D.; RODRIGUES, C. Efeito dos tributos no custo de produção, na rotação e na reforma de Eucalyptus spp. Cerne, Lavras, v. 11, n. 1, p. 70-83, 2005.

SILVA, C. S. J. Análise econômica da produção de madeira de candeia (Eremanthus erythropappus) em plantios. 2011. 135 f. Dissertação (Mestrado em Engenharia Florestal) - Universidade Federal de Lavras, Lavras, 2011.

SILVA, S. C. et al. Economic viability of cerrado vegetation management under conditions of risk. Cerne, Lavras, v. 17, n. 2, p. 141-149, 2011.

SOUZA, J. L. M. Modelo para a análise de risco econômico aplicado ao planejamento de projetos de irrigação para cultura do cafeeiro. 2001. 253 f. Tese (Doutorado em Agronomia) - Escola Superior de Agricultura "Luiz de Queiroz", Piracicaba, 2001. 